\title{
miR-654-3p suppresses cell viability and promotes apoptosis by targeting RASAL2 in non-small-cell lung cancer
}

\author{
JIE XIONG ${ }^{1}$, SHIGANG XING $^{1}$, ZHENG DONG $^{1}$, LEI NIU $^{1}$, QINGHUA XU $^{1}$, \\ YUSHENG LI ${ }^{1}$, PINGYI LIU ${ }^{2}$ and PEIXIA YANG ${ }^{1}$
}

Departments of ${ }^{1}$ Respiratory Medicine and ${ }^{2}$ Clinical Laboratory, Linyi Central Hospital, Linyi, Shandong 276400, P.R. China

Received March 9, 2018; Accepted November 9, 2018

DOI: $10.3892 / \mathrm{mmr} .2020 .11763$

\begin{abstract}
Non-small-cell lung cancer (NSCLC) accounts for $80 \%$ of lung cancer cases, and is the leading cause of cancer-associated mortality worldwide. The present study aimed to investigate the roles of microRNA (miR)-654-3p in NSCLC. The expression levels of miR-654-3p and its target ras protein activator like 2 (RASAL2) mRNA were determined by reverse transcription-quantitative polymerase chain reaction; protein expression was analyzed by western blotting. Plasmids expressing miR-654-3p mimics were constructed and transfected into A549 cells. In addition, the viability and apoptotic rate of cells were analyzed by an MTT assay and flow cytometry, respectively. A luciferase reporter assay was performed to verify whether RASAL2 is a target of miR-654-3p. Downregulated miR-654-3p and upregulated RASAL2 expression were observed in tumor tissues and cells. Cell viability was suppressed and the apoptotic rate was increased in the miR-654-3p mimics-transfected cells compared with the control. Luciferase activity was decreased in the RASAL2-3' untranslated region-wild type group treated with miR-654-3p mimics. Furthermore, the present study revealed that overexpression of miR-654-3p could suppress the viability and induce the apoptosis of cells by targeting RASAL2 in NSCLC. The present findings may contribute to developments in the treatment of NSCLC.
\end{abstract}

\section{Introduction}

Non-small-cell lung cancer (NSCLC) is a disease in which malignant cells proliferate in the tissues of the lung. It accounts for $\sim 85 \%$ of all lung cancer cases, and is the

Correspondence to: Dr Peixia Yang, Department of Respiratory Medicine, Linyi Central Hospital, 17 Jiankang Road, Linyi, Shandong 276400, P.R. China

E-mail: dr_peixiayang@163.com

\section{Abbreviation: NSCLC, non-small-cell lung cancer}

Key words: miR-654-3p, non-small-cell lung cancer, ras protein activator like 2 , proliferation, apoptosis leading cause of cancer-associated mortality worldwide (1). There are several types NSCLC depending on the type of cancer cell, including squamous cell carcinoma (squamous cell origin), large cell carcinoma (numerous types of undifferentiated cells) and adenocarcinoma (cells that line the alveoli) (2). It is well known that smoking is a major risk factor for the development of NSCLC (3). Additionally, there are numerous modes of NSCLC treatment, including surgery, radiation therapy, chemotherapy, targeted therapy, laser therapy, photodynamic therapy, cryosurgery and radiosensitizers (4); however, due to limitations in diagnosis, the majority of patients with NSCLC are diagnosed at stages III and IV, for whom existing treatments are not curative (5). Therefore, there is an urgent need for more efficient therapeutic approaches for NSCLC treatment.

MicroRNAs (miRNAs) are a group of small non-coding RNAs comprising 22-24 nucleotides (6). miRNAs specifically bind to the 3' untranslated region (3' UTR) of target mRNAs, leading to mRNA degradation and abnormal levels of protein expression (7). miRNAs are differentially expressed in tumor and normal tissues, and function as oncogenes or tumor suppressors (8). Numerous miRNAs have been reported to serve key roles in the pathogenesis of NSCLC (9). miR-34b and miR-520h have been suggested to be key factors in the regulation of NSCLC, and miR-22 has been reported as an oncogene and novel biomarker $(9,10)$. The tumor suppressor properties of miR-654-3p have been investigated in papillary thyroid cancer and miR-654-3p has also been suggested as a potential biomarker for the tumorigenicity of VERO cells $(11,12)$. It has been demonstrated that the expression of miR-654-3p differed in NSCLC tissues from normal tissues (9).

Ras protein activator like 2 (RASAL2) gene encodes the enzyme, RasGTPase-activating protein nGAP (13). RasGTPases are essential components of signaling pathways that propagate signals from cell surface receptors to regulate a variety of cellular processes, including cell cycle progression, cell survival, actin cytoskeletal organization, cell polarity and movement (14). It was recently demonstrated that RASAL2 serves roles as a tumor and metastasis suppressor by inhibiting the proliferative and metastatic abilities of nasopharyngeal carcinoma cells (15).

In the present study, bioinformatics research using TargetScan, revealed that RASAL-2 was a target of miR-654-3p. The present study aimed to investigate the effects of miR-654-3p 
on the viability and apoptosis of NSCLC cells by targeting RASAL2, which may have potential as a novel therapeutic target for the treatment of NSCLC.

\section{Materials and methods}

Patients and tissues. Tumor tissues were collected from 45 patients with NSCLC between August 2016 and August 2017 at Linyi Central Hospital (Linyi, China). The clinicopathological features (including gender, age, tumour stage, and tumor, node and metastasis staging) of patients with NSCLC enrolled in our study were presented in Table I. The tumor and the paracancerous tissues were fixed in $10 \%$ formalin for $48 \mathrm{~h}$ at room temperature. Tumor and non-tumor samples were confirmed by pathological examination. No patients received chemotherapy or radiotherapy prior to surgery. In addition, the present study was approved by the ethics committee of Linyi Central Hospital, and written informed consent was obtained from all patients prior to enrolment.

Cell line. A549 cells were purchased from the Shanghai Institute of Biochemistry and Cell Biology (Shanghai, China) and cultured in RPMI-1640 medium (HyClone; GE Healthcare Life Sciences, Logan, UT, USA) with 10\% fetal bovine serum (Gibco; Thermo Fisher Scientific, Inc., Waltham, MA, USA) at $37^{\circ} \mathrm{C}$ in $5 \% \mathrm{CO}_{2}$ in a humidified incubator.

Cell transfection. The cells were divided into three groups: Control, negative control (NC) and miR-654-3p mimics. The cells in the control group were incubated in medium without treatment; cells in the NC group were transfected with miR-NC mimics, and cells in miR-654-3p mimics group were transfected with miR-654-3p mimics. All cells were incubated at $37^{\circ} \mathrm{C}$ in a humidified $5 \% \mathrm{CO}_{2}$ atmosphere for $48 \mathrm{~h}$. The miR-654 mimics and control were obtained from New England Biolabs, Inc. (Ipswich, MA, USA). The cells were seeded in triplicate in 24-well plates and transfected with $500 \mathrm{ng}$ miR-654-3p mimics or miR-NC mimics using $2.5 \mu \mathrm{l}$ Lipofectamine ${ }^{\circledR} 2000$ (Thermo Fisher Scientific, Inc.). After $6 \mathrm{~h}$ post-transfection, the medium was replaced with fresh medium containing 10\% FBS. The sequences of the transfected mimics were the following: miR-654 mimics forward, 5'-UGGUGGGCCGCAGAACAUGUGC-3'; miR-654 mimics reverse, 5'-ACAUGUUCUGCGGCCCACGAAU-3'; NC mimics forward, 5'-UUCUCCGAACGUGUCACGUUU-3'; NC mimics reverse, 5'-ACGUGACACGUUCGGAGAAUU-3'.

Reverse transcription-quantitative polymerase chain reaction ( $R T-q P C R)$. RT-qPCR was employed to determine miR-654-3p and mRNA expression levels. An RNeasy Mini kit (Qiagen GmbH, Hilden, Germany) was used to isolate RNA from cells or tissues according to the manufacturer's protocol, and theconcentration of RNA was determined using a NanoDrop 2000 spectrophotometer (NanoDrop; Thermo Fisher Scientific, Inc., Wilmington, DE, USA). An M-MLV reverse transcriptase cDNA synthesis kit (Thermo Fisher Scientific) was applied to synthesize cDNA via RT; samples were incubated at $43^{\circ} \mathrm{C}$ for $30 \mathrm{~min}, 97^{\circ} \mathrm{C}$ for $5 \mathrm{~min}$ and $5^{\circ} \mathrm{C}$ for $5 \mathrm{~min}$. The PrimeScript ${ }^{\mathrm{TM}}$ RT-PCR kit (Sigma-Aldrich; Merck KGaA, Darmstadt, Germany) was used for qPCR. The thermocycling conditions were as follows: $95^{\circ} \mathrm{C}$ for $6 \mathrm{~min}$ (denaturation), $94^{\circ} \mathrm{C}$ for $30 \mathrm{sec}$ (initiation), $60^{\circ} \mathrm{C}$ for $30 \mathrm{sec}$ (annealing), and $75^{\circ} \mathrm{C}$ for $1.5 \mathrm{~min}$ (elongation) for 36 cycles. U6 was used to normalize the expression of miR-654-3p and GAPDH was used as an internal reference for mRNA expression. This experiment was performed in triplicate. Expression was quantified using the $2^{-\Delta \Delta C q}$ method (16). The sequences of the primers used are as follows: miR-654-3p, forward: 5'-GGGATGTCTGCTGACCA-3'; reverse: 5'-CAGTGC GTGTCGTGGA-3'; U6, forward: 5'-CTCGCTTCGGCA GCACA-3', reverse: 5'-AACGCTTCACGAATTTGCGT-3'; Bcl-2-associated x protein (Bax), forward: 5'-CACCAGCTC TGAACAGATCATGA-3', reverse: 5'-TCAGCCCATCTT CTTCCAGATGT-3'; B cell lymphoma-2 (Bcl-2), forward: 5'-CACCCCTGGCATCTTCTCCTT-3', reverse: 5'-AGC GTCTTCAGAGACAGCCAG-3'; RASAL2, forward: 5'-TGT TCTGTCCTTGAGCCAGT-3', reverse: 5'-TCCACCTCA GACATCACCAA-3'; and GAPDH, forward: 5'-GCACCA CCAACTGCTTAGC-3', reverse: 5'-GGCATGGACTGT GGTCATGAG-3'.

Western blotting. Protein expression in tissues and cells was investigated by western blotting. Cells and tissue samples were lysed in radioimmunoprecipitation assay buffer (Thermo Fisher Scientific, Inc.) and the total protein concentration was determined using a Bicinchoninic Acid assay (Thermo Fisher Scientific, Inc.). GAPDH was used as a loading control; $2 \mu \mathrm{g}$ protein was loaded for $15 \%$ SDS-PAGE, and then transferred to a polyvinylidene fluoride membrane (Thermo Fisher Scientific, Inc.). The membrane was blocked with 5\% skimmed milk for $2 \mathrm{~h}$ at $37^{\circ} \mathrm{C}$, then incubated with the following primary antibodies: Anti-RASAL-2 (cat. no. ab216127; 1:5,000; Abcam, Cambridge, UK), anti-Bcl-2 (cat. no. ab209039; 1:5,000; Abcam), anti-Bax (cat. no. ab32503; 1:5,000; Abcam) and anti-GAPDH (cat. no. ab181602; 1:10,000; Abcam) for $1 \mathrm{~h}$ at room temperature. The membrane was then incubated with a horseradish peroxidase-conjugated anti-rabbit antibody (ab6721; 1:5,000; Abcam) for $45 \mathrm{~min}$ at room temperature. Subsequently, an enhanced chemiluminescence western blotting kit (Pierce; Thermo Fisher Scientific, Inc.) was used to visualise the proteins. The gray values were obtained using Image J image analysis software (National Institutes of Health, Bethesda, MD, USA). This experiment was performed in triplicate.

MTT assay. Transfected cells were seededinto 24-well plates $\left(2 \times 10^{6}\right.$ cells $\left./ \mathrm{ml}\right)$ and incubated at $37^{\circ} \mathrm{C}$ with $5 \% \mathrm{CO}_{2}$. Subsequently, an MTT Cell Viability Assay kit (Sigma-Aldrich; Merck KGaA) was used, according to the manufacturer's protocols. The optical density was measured at a wavelength of $570 \mathrm{~nm}$ using a microplate reader at $0,12,24$ and $48 \mathrm{~h}$. The experiment was conducted in triplicate.

Flow cytometry assay. Transfected cells were plated into 24-well plates $\left(2 \times 10^{6}\right.$ cells $\left./ \mathrm{ml}\right)$ and incubated at $37^{\circ} \mathrm{C}$ with $5 \% \mathrm{CO}_{2}$ for $72 \mathrm{~h}$. Then, the cells were treated with propidium iodide (10 $\mu \mathrm{g} / \mathrm{ml}$; Sigma-Aldrich; Merck KGaA) and Annexin V-fluorescein isothiocyanate $(50 \mu \mathrm{g} / \mathrm{ml}$; Becton, Dickinson and Company, Franklin Lakes, NJ, USA) in the dark for $15 \mathrm{~min}$ at room temperature. Subsequently, the 


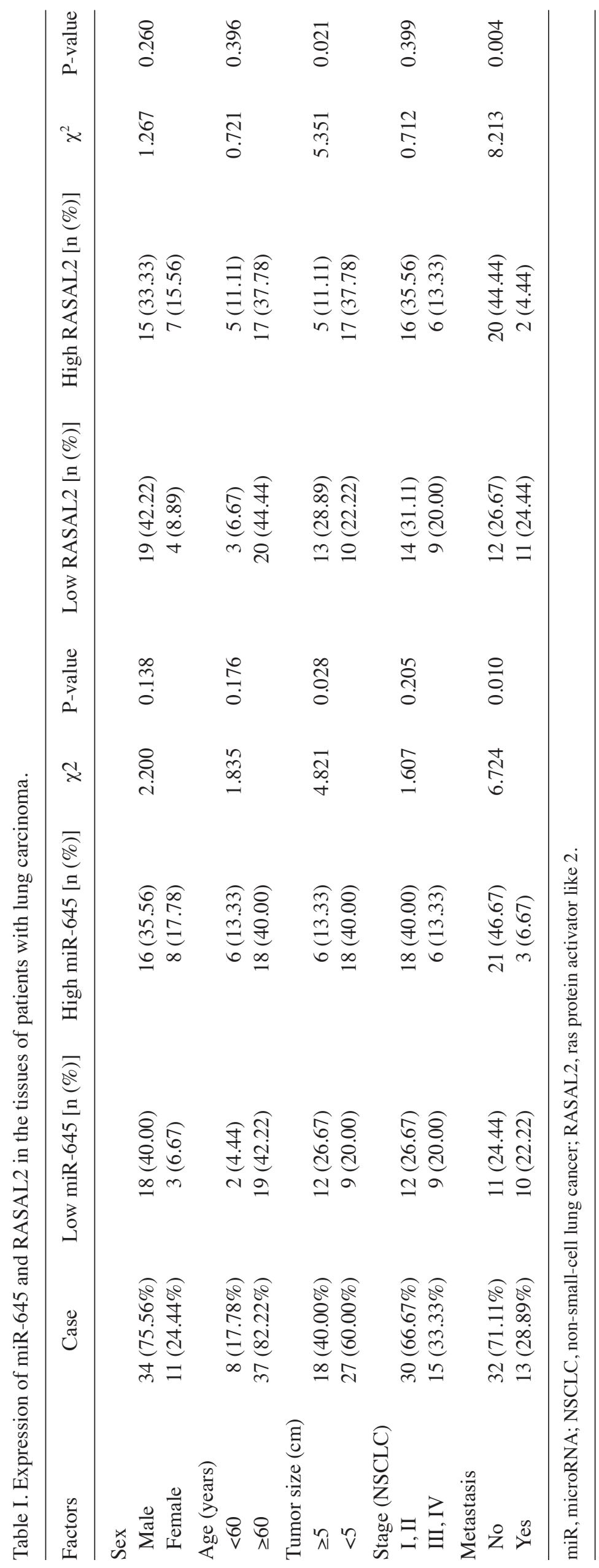


A

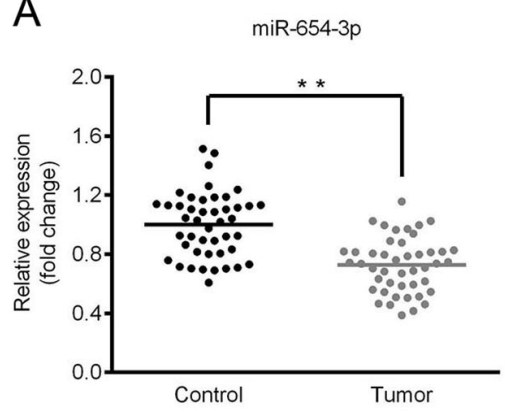

B

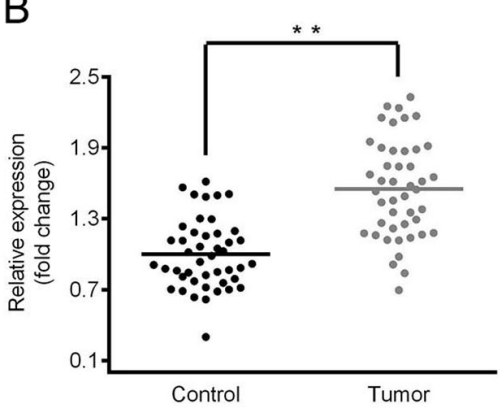

C

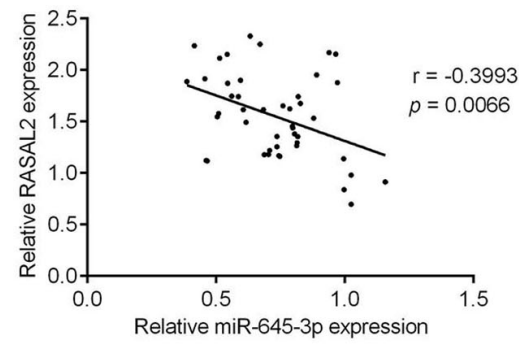

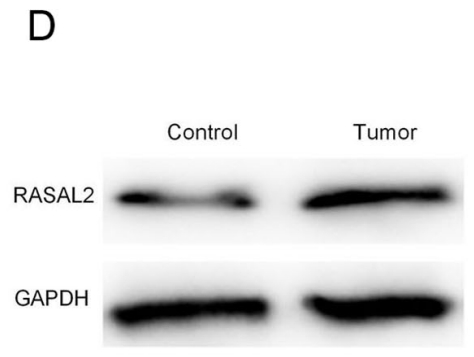

E

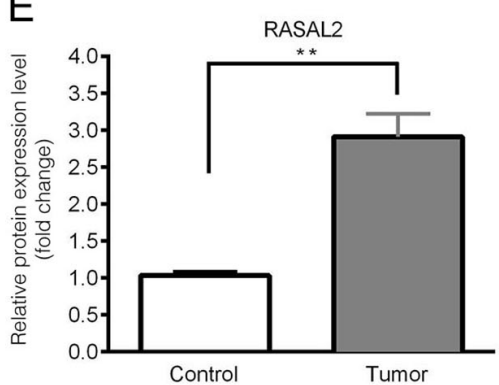

Figure 1. Downregulated miR-654-3p expression and upregulated RASAL2 expression in lung cancer tissues. The expression of miR-65-3p and RASAL2 was determined by reverse transcription-quantitative polymerase chain reaction and western blotting. (A) Expression of miR-654-3p in tumor tissues. (B) Expression of RASAL2 in tumor tissues. (C) Negative correlation was observed between the expression of miR-654-3p and RASAL2. (D) Protein expression levels of RASAL2. (E) Quantitative analysis of D. ${ }^{* *} \mathrm{P}<0.01$. miR, microRNA; RASAL2, ras protein activator like 2.

stained cells were analyzed using a FACScanflow cytometer (Becton, Dickinson and Company) and Diva software (version 8.0; Becton, Dickinson and Company)to determine the apoptotic rate.

Bioinformatics analysis and luciferase reporter assay. Bioinformatics analysis using TargetScan 7.1 (http://www. targetscan.org/vert_71/) revealed that RASAL2 was a target of miR-654-3p. A luciferase reporter assay was performed to verify whether RASAL2 was a direct target of miR-654-3p. The cells were seeded in 96-well plates $\left(2 \times 10^{3}\right.$ cells/well $)$ and incubated at $37^{\circ} \mathrm{C}$ with $5 \% \mathrm{CO}_{2}$ for $24 \mathrm{~h}$. Subsequently, RASAL2-3'UTR-wild type (WT) and RASAL2-3'UTR-mutant (MUT) plasmids $(2.5 \mu \mathrm{g}$, Addgene, Inc., Cambridge, MA, USA), miR-654-3p mimics and miR-NC were transfected to cells using 2.5 $\mu$ 1 Lipofectamine 2000. A BioLux ${ }^{\circledR}$ Gaussia Luciferase Reporter Assay kit (New England Biolabs, Inc.) was utilized for the analysis of luciferase activity, according to the manufacturer's protocols. Luciferase activity was normalized to that of Renilla luciferase.

Statistical methods. All data are presented as the mean \pm standard deviation and were analyzed using GraphPad Prism version 5.01 (GraphPad Software, Inc., La Jolla, CA, USA). A paired Student's t-test was used to examine differences between the two groups. An unpaired Student's t-testwas used to compare the luciferase activity between $\mathrm{NC}$ and miR-654-3p groups. One-way analysis of variance followed by a Newman-Keuls post-hoc test was used to examine differences among three groups. The correlation between miR-654-3p and RASAL2 was analysed using Pearson's

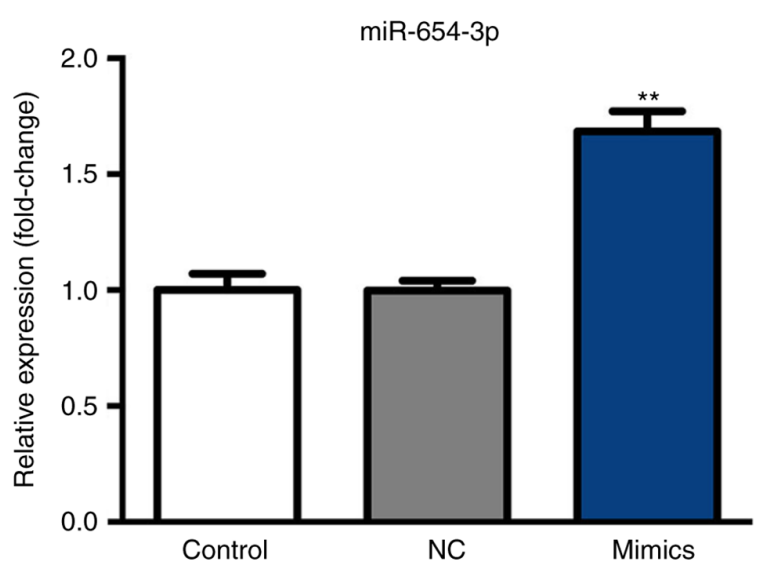

Figure 2. Expression of miR-654-3p following transfection with miR-654-3p mimics. Expression of $\mathrm{mR}-654-3 \mathrm{p}$ was determined by reverse transcription-quantitative polymerase chain reaction following transfection and incubation at $37^{\circ} \mathrm{C}$ in $5 \% \mathrm{CO}_{2} .{ }^{* *} \mathrm{P}<0.01$ vs. the control groups. Control, untreated group; $\mathrm{NC}$, negative control group; miR, microRNA; mimics, cells transfected with miR-654-3p.

correlation coefficient. A $\chi^{2}$ test was performed to analyze the data presented in Table I. $\mathrm{P}<0.05$ was considered to indicate a statistically significant difference.

\section{Results}

Downregulated expression of miR-654-3p and upregulated expression of RASAL2 in lung cancer tissues. The expression levels of miR-654-3p and RASAL2 were determined by 

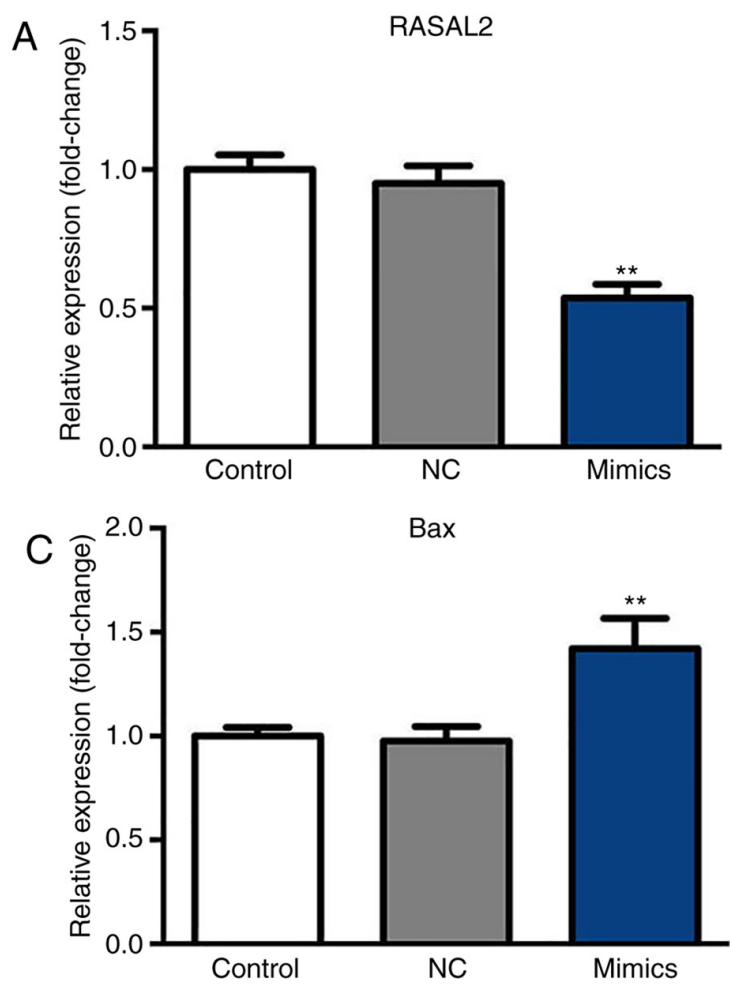

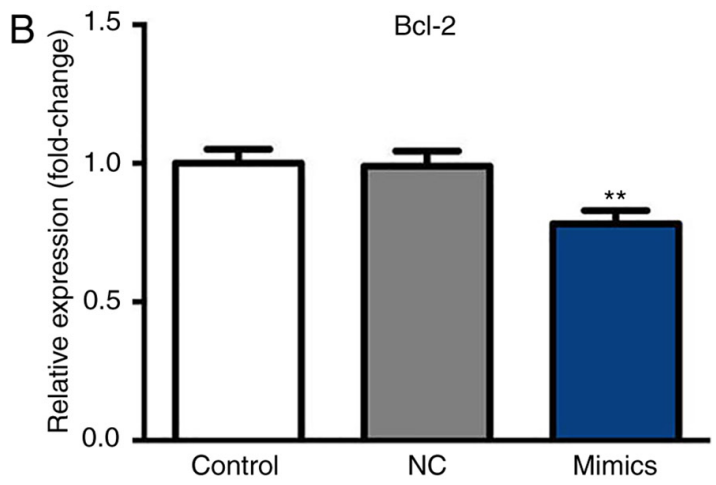

D

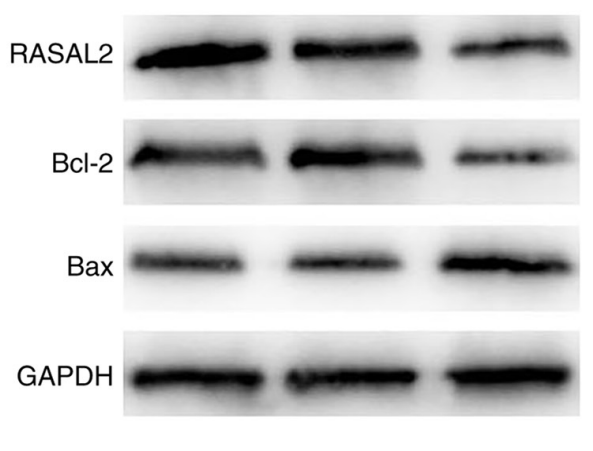

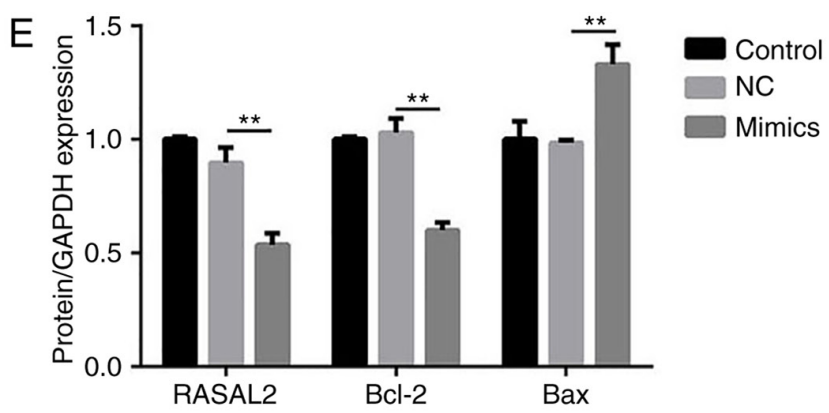

Figure 3. Suppressed RASAL2 and Bcl-2 expression and upregulated Bax expression in A549 cells following transfection with miR-654-3p mimics. The mRNA and protein expression levels were analyzed by RT-qPCR and western blotting following transfection with miR-654-3p mimics for $48 \mathrm{~h}$. Expression of (A) RASAL2, (B) Bcl-2 and (C) Bax following transfection with mimics. (D) Protein expression levels of RASAL2, Bcl-2 and Bax. (E) Statistical data were presented. ${ }^{* *} \mathrm{P}<0.01$ vs. the control groups. Bcl-2, B cell lymphoma-2; Bax, Bcl-2-associated X protein; Control, untreated group; NC, negative control group; miR, microRNA; Mimics, cells transfected with miR-654-3p; RASAL2, ras protein activator like 2.

RT-qPCR and western blotting. As presented in Fig. 1A and B, significantly downregulated expression of miR-654-3p and upregulated expression of RASAL2 were observed in the tumor tissues compared with control. A negative correlation between miR-654-3p and RASAL2 expression was identified (Fig. 1C). Furthermore, the expression of RASAL2 protein was notably upregulated in tumor tissues compared with in the control (Fig. 1D and E). In addition, the expression levels of miR-654-3p and RASAL2 were significantly associated with the clinicopathological features of NSCLC, including tumor size and lymph node metastasis (Table I).

Expression of miR-654-3p following transfection with miR-654-3p mimics. After $6 \mathrm{~h}$ following transfection with miR-654-3p mimics and a 48 -h incubation at $37^{\circ} \mathrm{C}$, miR-654-3p expression was analyzed by RT-qPCR. As presented in Fig. 2, miR-654-3p expression was significantly upregulated in the mimics group compared with the control groups.

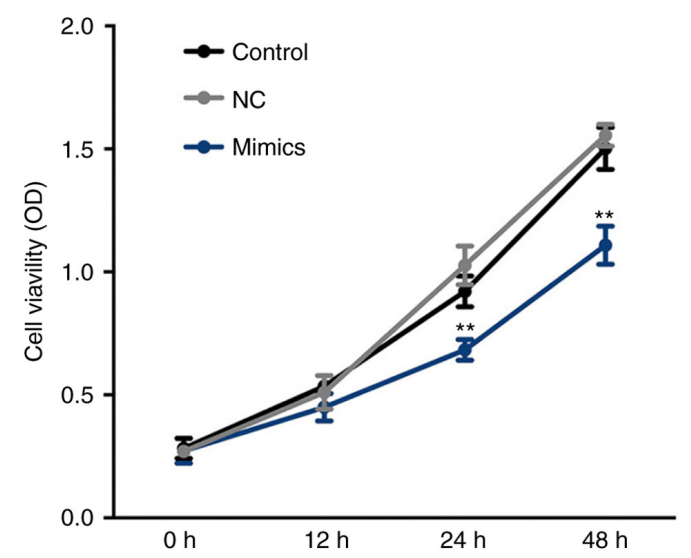

Figure 4. Suppressed viability of A549 cells following transfection with miR-654-3p mimics. An MTT assay was performed at 0, 12, 24 and $48 \mathrm{~h}$ pot-transfection at $37^{\circ} \mathrm{C}$ in $5 \% \mathrm{CO}_{2} .{ }^{* * *} \mathrm{P}<0.01$ vs. the control groups. Control, untreated group; NC, negative control group; miR, microRNA; Mimics, cells transfected with miR-654-3p. 

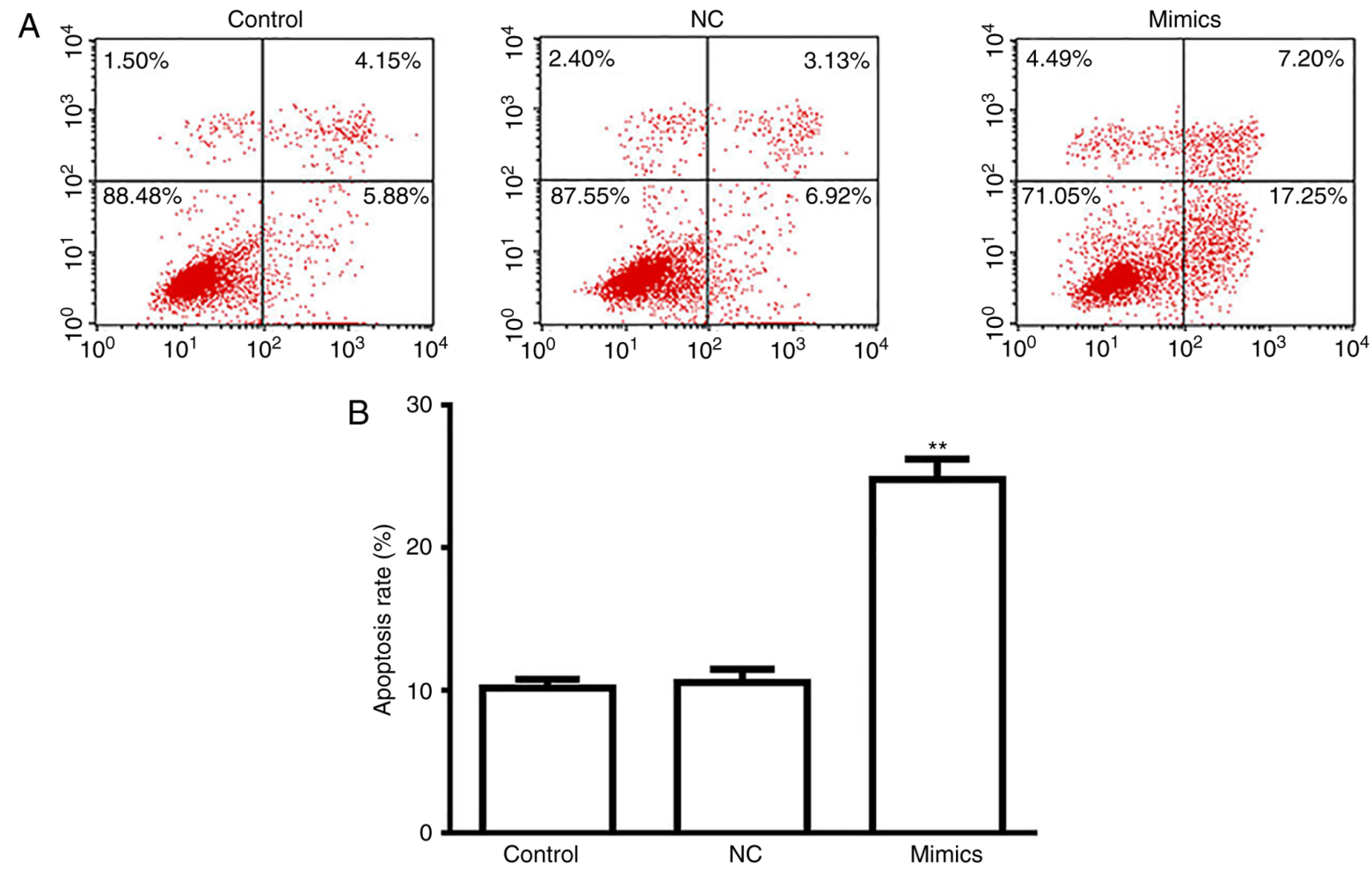

Figure 5. Apoptosis of A549 cells is induced following transfection with miR-654-3p mimics. After $48 \mathrm{~h}$ incubation at $37^{\circ} \mathrm{C}$ with $5 \% \mathrm{CO}_{2}$, cells were stained with propidium iodide and Annexin V-fluorescein isothiocyanate. (A) Apoptosis of A549 cells via flow cytometery assay. (B) Quantification of A. **P $<0.01$ vs. the control groups. Control, untreated group; NC, negative control group; miR, microRNA; Mimics, cells transfected with miR-654-3p.

A Position 1434-1440 of RASAL2 3'UTR

hsa-miR-654-3p

Mutant position of RASAL2 3'UTR

B

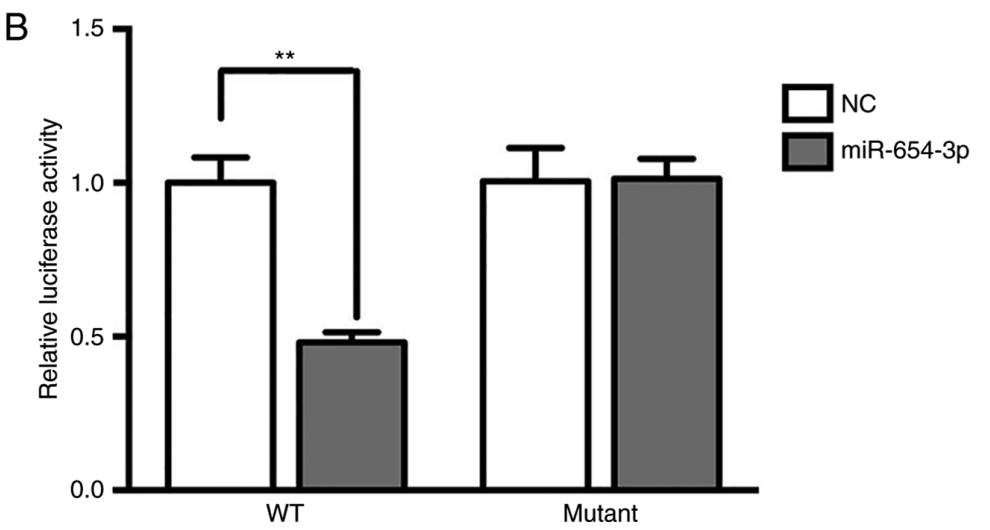

$5^{\prime} \quad$... AGGUAACAUGUUAAACAGACAUU

\| | || ||

3' UUCCACUACCAGUCGUCUGUAU

$5^{\prime}$... UUCCACUACCAGUCGGACACCU

Figure 6. Target verification by luciferase reporter assay. (A) Bioinformatics target prediction using TargetScan. (B) Relative luciferase activity following transfection. Control, untreated group; NC, negative control group; Mimics, cells transfected with miR-654-3p. ${ }^{* *} \mathrm{P}<0.01$.

Suppressed RASAL2 and Bcl-2 expression, and upregulated Bax expression in A549 cells following transfection with miR-654-3p mimics. After $48 \mathrm{~h}$ incubation at $37^{\circ} \mathrm{C}$ with $5 \% \mathrm{CO}_{2}$, the expression levels of RASAL2, Bax and Bcl-2 were determined by RT-qPCR and western blotting. As presented in Fig. 3A and B, the mRNA expression levels of RASAL2 and Bcl-2 were significantly decreased in the miR-654-3p mimics group compared with the control groups, respectively. Furthermore, the mRNA expression levels of Bax were significantly upregulated in the mimics group compared with the control groups (Fig. 3C). Additionally, compared with the NC group, significantly decreased expression of RASAL2 
and Bcl-2, and increased expression of Bax protein were observed in response to miR-654-3p transfection (Fig. 3D and E). Therefore, miR-654-3p may inhibit the expression of RASAL2 and Bcl-2 expression, while inducing that of Bax.

Suppressed viability of A549 cells following transfection with miR-654-3p mimics. Cell viability was determined at 0, 12 , 24 and 48 h. As presented in Fig. 4, cell viability was significantly inhibited in the miR-654-3p mimics group compared with the control groups at 24 and $48 \mathrm{~h}$. These results suggest that cell viability is suppressed by miR-654-3p upregulation.

A549 cell apoptosis is induced following transfection with miR-654-3p mimics. As presented in Fig. 5, a significantly increased rate of apoptosis was observed in the miR-654-3p mimics group compared with the control groups (Fig. 5A and B). Thus, overexpression of miR-654-3p may induce apoptosis by targeting RASAL2.

Target verification by luciferase reporter assay. Bioinformatics analysis using TargetScan suggested that RASAL2 was a target of miR-654-3p (Fig. 6A). Additionally, the luciferase activity was significantly decreased in the RASAL2-3'UTR-WT group treated with miR-654-3p mimics compared with the control (Fig. 6B), which indicated that RASAL2 was a target of miR-654-3p.

\section{Discussion}

In the present study, it was suggested that RASAL2 was a target of miR-654-3p in NSCLC as predicted by TargetScan analysis and demonstrated via a luciferase activity assay (17). Overexpression of miR-654-3p may inhibit the expression of RASAL2 and Bcl-2, while inducing that of Bax.

The present study aimed to investigate the effects of miR-645-3p on the viability and apoptosis of NSCLC cells. The results indicated that miR-654-3p expression was downregulated in tumor tissues and NSCLC cells, which was also reported by $\mathrm{Xu}$ et al (9). Furthermore, bioinformatics and luciferase reporter analyses revealed RASAL2 as a target of miR-654-3p in the present study. It was also suggested that the upregulated expression of RASAL2 in A549 cells was reversed by overexpression of miR-654-3p, which resulted in reduced RASAL 2 and Bcl-2 expression, and increased Bax expression, and further resulted in the suppression of cell viability and the induction of apoptosis of A549 cells. These results indicated that miR-654-3p was associated with RASAL2 expression, and the viability and apoptosis of NSCLC cells.

RASAL2 has been studied in breast cancer, hepatocellular carcinoma and colorectal cancer, among others; however, its role in NSCLC requires further investigation (18-20). Yan et al (21) reported that miR-136 acts as a tumor suppressor by targeting RASAL2, thus inhibiting cell invasion and metastasis in triple-negative breast cancer. miR-136 was also demonstrated to be an oncogene in human NSCLC (21). Therefore, the combined effect of numerous miRNAs on the regulation of RASAL2 remains to be elucidated. Furthermore, RASAL2 is a member of the RAS GTPase-activating proteins, which catalyzes the dephosphorylation of GTP into GDP, inactivating Ras $(14,22)$. RASAL2 was proposed to affect epithelial-mesenchymal transition via the mitogen-activated protein kinase/SRY-box 2 pathway, which contributes to alterations in the migration and invasion of breast and lung cancer cells $(13,23)$. Therefore, investigations into the effects of miR-654-3p on cell migration and invasion are required to fully characterize the roles of miR-654-3p of A549 in NSCLC.

In addition, Geraldo et al (11) revealed that $14 \mathrm{q} 32$-encoded miRNAs, including miR-495-3p, miR-654-3p, miR-376a-3p and miR-487b-3p, could function as tumor suppressor genes when their expression was downregulated. Therefore, future studies should investigate the potential of these miRNAs in the treatment of NSCLC. In the present study, it was demonstrated that RASAL2 was a target of miR-654-3p in NSCLC; however, the specific mechanism underlying the effects of miR-654-3p in the cell cycle, proliferation, migration, invasion and apoptosis should be investigated beyond the RASAL2 pathway. Additionally, although the number of patients involved in the present study was small, future investigations can be conducted using greater patient cohorts.

In conclusion, the present study reported that miR-139-5p is downregulated and RASAL2 is upregulated in NSCLC. Overexpression of miR-139-5p could suppress the viability and promote the apoptosis of NSCLC cells by targeting RASAL2. Therefore, miR-139-5p may act as a potential therapeutic target for the treatment of NSCLC.

\section{Acknowledgements}

Not applicable.

\section{Funding}

No funding was received.

\section{Availability of data and materials}

The datasets used and/or analysed during the present study are available from the corresponding author on reasonable request.

\section{Authors' contributions}

JX drafted the manuscript. JX,SX, ZD, YL and PL collected and analyzed the data. JX ,SX, ZD, YL, PL, LN and QX performed the experiments. PY designed the study. All authors read and approved the final manuscript.

\section{Ethics approval and consent to participate}

The present study was approved by The Ethics Committee of Linyi Central Hospital. Written informed consent was obtained from all patients prior to enrolment.

\section{Patient consent for publication}

Not applicable.

\section{Competing interests}

The authors declare that they have no competing interests. 


\section{References}

1. Huang G, Sun X, Liu D, Zhang Y, Zhang B, Xiao G, Li X, Gao X $\mathrm{Hu} \mathrm{C}$, Wang M, et al: The efficacy and safety of anti-PD-1/PD-L1 antibody therapy versus docetaxel for pretreated advanced NSCLC: A meta-analysis. Oncotarget 9: 4239-4248, 2018.

2. Quintanal-Villalonga Á, Ojeda-Márquez L, Marrugal Á, Yagüe P, Ponce-Aix S, Salinas A, Carnero A, Ferrer I, Molina-Pinelo S and Paz-Ares L: The FGFR4-388arg variant promotes lung cancer progression by N-cadherin induction. Sci Rep 8: 2394, 2018.

3. Gouvinhas C, De Mello RA, Oliveira D, Castro-Lopes JM, Castelo-Branco P, Dos Santos RS, Hespanhol V and Pozza DH: Lung cancer: A brief review of epidemiology and screening. Future Oncol 14: 567-575, 2018.

4. Rios J, Gosain R, Goulart BH, Huang B, Oechsli MN, McDowell JK, Chen Q, Tucker T and Kloecker GH: Treatment and outcomes of non-small-cell lung cancer patients with high comorbidity. Cancer Manag Res 10: 167-175, 2018.

5. Song YJ, Gao XH, Hong YQ and Wang LX: Direct bilirubin levels are prognostic in non-small cell lung cancer. Oncotarget 9: 892-900, 2018

6. Lan H, Lu H, Wang $\mathrm{X}$ and Jin H: MicroRNAs as potential biomarkers in cancer: Opportunities and challenges. Biomed Res Int 2015: 125094, 2015

7. Van Schooneveld E, Wildiers H, Vergote I, Vermeulen PB, Dirix LY and Van Laere SJ: Dysregulation of microRNAs in breast cancer and their potential role as prognostic and predictive biomarkers in patient management. Breast Cancer Res 17: 21, 2015.

8. Zhang XY, Liu DJ, Yuan RB, Zhang DH, Li SR, Zhang SH and Zhang LY: Low expression of miR-597 is correlated with tumor stage and poor outcome in breast cancer. Eur Rev Med Pharmacol Sci 22: 456-460, 2018.

9. $\mathrm{Xu} \mathrm{C}$, Zheng $\mathrm{Y}$, Lian $\mathrm{D}$, Ye S, Yang J and Zeng Z: Analysis of microRNA expression profile identifies novel biomarkers for non-small cell lung cancer. Tumori 101: 104-110, 2015.

10. Shi GL, Chen Y, Sun Y, Yin YJ and Song CX: Significance of serum microRNAs in the auxiliary diagnosis of non-small cell lung cancer. Clin Lab 63: 133-140, 2017.

11. Geraldo MV, Nakaya HI and Kimura ET: Down-regulation of 14q32-encoded miRNAs and tumor suppressor role for miR-654-3p in papillary thyroid cancer. Oncotarget 8: 9597-9607, 2017.

12. Teferedegne B, Macauley J, Foseh G, Dragunsky E, Chumakov K, Murata H, Peden K and Lewis AM Jr: MicroRNAs as potential biomarkers for VERO cell tumorigenicity. Vaccine 32: 4799-4805, 2014.
13. Hui K, Gao Y, Huang J, Xu S, Wang B, Zeng J, Fan J, Wang X, Yue Y, Wu S, et al: RASAL2, a RAS GTPase-activating protein, inhibits stemness and epithelial-mesenchymal transition via MAPK/SOX2 pathway in bladder cancer. Cell Death Dis 8: e2600, 2017.

14. Maertens $\mathrm{O}$ and Cichowski $\mathrm{K}$ : An expanding role for RAS GTPase activating proteins (RAS GAPs) in cancer. Adv Biol Regul 55: 1-14, 2014.

15. Wang Z, Wang J, Su Y and Zeng Z: RASAL2 inhibited the proliferation and metastasis capability of nasopharyngeal carcinoma. Int J Clin Exp Med 8: 18765-18771, 2015.

16. Livak KJ and Schmittgen TD: Analysis of relative gene expression data using real-time quantitative PCR and the 2(-Delta DeltaC(T)) method. Methods 25: 402-408, 2001.

17. Fang JF, Zhao HP, Wang ZF and Zheng SS: Upregulation of RASAL2 promotes proliferation and metastasis, and is targeted by miR-203 in hepatocellular carcinoma. Mol Med Rep 15: 2720-2726, 2017.

18. Olsen SN, Wronski A, Castaño Z, Dake B, Malone C, De Raedt T, Enos M, DeRose YS, Zhou W, Guerra S, et al: Loss of RasGAP tumor suppressors underlies the aggressive nature of luminal B breast cancers. Cancer Discov 7: 202-217, 2017.

19. Jia Z, Liu W, Gong L and Xiao Z: Downregulation of RASAL2 promotes the proliferation, epithelial-mesenchymal transition and metastasis of colorectal cancer cells. Oncol Lett 13: 1379-1385, 2017.

20. Shen H, Wu X, Zhang Y, Deng G, Ma J, Qu Y and Zeng S: Expression of RASAL2 in hepatocellular carcinoma and the clinical significance. Zhong Nan Da Xue Xue Bao Yi Xue Ban 40: 250-255, 2015 (In Chinese)

21. Yan M, Li X, Tong D, Han C, Zhao R, He Y and Jin X: miR-136 suppresses tumor invasion and metastasis by targeting RASAL2 in triple-negative breast cancer. Oncol Rep 36: 65-71, 2016.

22. Noto S, Maeda T, Hattori S, Inazawa J, Imamura M, Asaka M and Hatakeyama M: A novel human RasGAP-like gene that maps within the prostate cancer susceptibility locus at chromosome 1q25. FEBS Lett 441: 127-131, 1998.

23. Li N and Li S: RASAL2 promotes lung cancer metastasis through epithelial-mesenchymal transition. Biochem Biophys Res Commun 455: 358-362, 2014.

This work is licensed under a Creative Commons Attribution-NonCommercial-NoDerivatives 4.0 International (CC BY-NC-ND 4.0) License. 